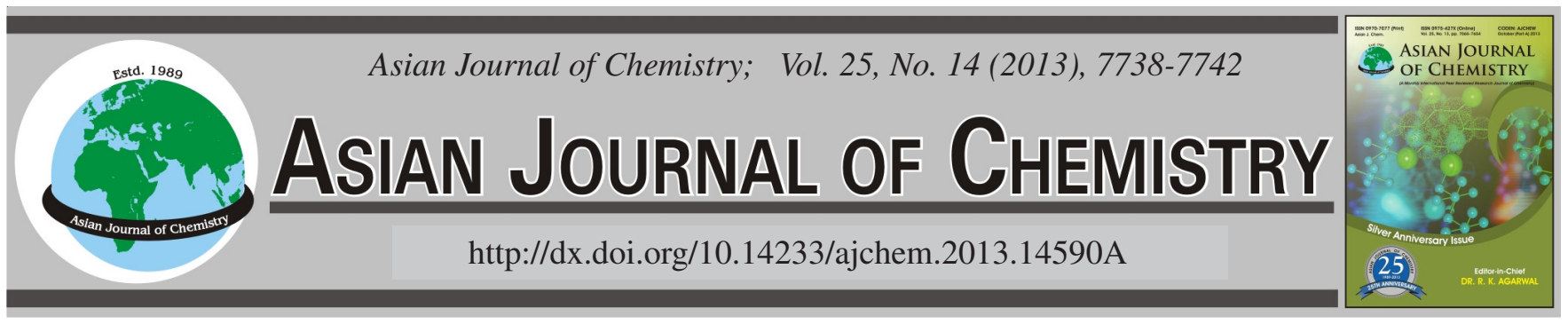

\title{
Synthesis of Novel Arylfurfurylchalcones
}

\section{Samina Aslam ${ }^{1}$, Nadia Asif ${ }^{2}$, Muhammad Naeem Khan ${ }^{3, *}$, Misbahul Ain Khan ${ }^{1}$, Munawar Ali Munawar ${ }^{2}$ and Muhammad Nasrullah ${ }^{1}$}

\begin{abstract}
${ }^{1}$ Department of Chemistry, The Islamia University of Bahawalpur, Bahawalpur, Pakistan
${ }^{2}$ Institute of Chemistry, University of the Punjab, Lahore, Pakistan

${ }^{3}$ Applied Chemistry Center, PCSIR Laboratories Complex, Lahore, Pakistan
\end{abstract}

*Corresponding author: E-mail: changwani_1@yahoo.com

(Received: 17 September 2012;

Various aryl furans-2-carbaldehyde chalcones with different acetophenones were prepared and characterized through their elemental analyses and spectroscopic techniques (FTIR, ${ }^{1} \mathrm{H}$ NMR, ${ }^{13} \mathrm{C}$ NMR and mass spectra).

Key Words: Aryl furans, Meerwein-arylation, Chalcones.

\section{INTRODUCTION}

Nitro group containing furans such as dantrolene, a muscle relaxant and nitrofurantoin an antibiotic are in clinical use ${ }^{1}$ while other derivatives find diverse applications in industry ${ }^{2-4}$. Yet other substituted furan-2-carbaldehydes possess a C 2 cabonyl group that may act as a reactive centre for various condensation reactions. Many of the published condensations products are biologically active compounds $s^{5,6}$ or can be used as intermediates in organic synthesis ${ }^{7,8}$.

Chalcones, $\alpha, \beta$-unsaturated ketones ${ }^{9-11}$ display a wide range of pharmaceutical properties, including cytotoxity ${ }^{12,13}$, antimitotic ${ }^{14}$, antimutagenic ${ }^{15}$ antitumor-promoting, antibacterial ${ }^{16}$, antiviral ${ }^{17}$ and anti-inflammatory ${ }^{18}$ activities. They are also useful in material science fields such as non-linear optics (NLO) $)^{19}$, optical limiting ${ }^{20}$, electrochemical sensing ${ }^{21}$, Langmuir films and photo-initiated polymerization ${ }^{22}$. Various chalcone derivatives are notable materials for their second harmonic generation $(\mathrm{SHG})^{23}$. They are well known intermediates for synthesizing various heterocyclic compounds. Cyclization of chalcones, leading to various heterocycles, such as thiazines, pyrimidines, pyrazoline etc. which have potentials as efficacious antibacterial agents. A survey of literature in the recent past reveals that some pyrazoline derivatives possess antibacterial ${ }^{24,25}$, anti-inflammatory ${ }^{26,27}$ and antifungal effects ${ }^{28}$.

Some 1-aryl-3-[5-( $p$-nitrophenyl)-2-propen-1-ones were obtained from the corresponding 5-p-nitrophenylfuran-2carbaldehyde are reported and were used for the synthesis of 1,2,4-triazolo[3,4-b]-1,3,4-thiadiazines claimed to have antibacterial and antiviral properties ${ }^{29}$. We would now like to report the synthesis of numerous new 1-aryl-3-arylfurylpropenes which are currently under investigation for their biological screening.

\section{EXPERIMENTAL}

All reagents and solvents were used as obtained from the supplier or recrystallized or redistilled as necessary. Thin layer chromatography was performed using aluminium sheets (Merck) coated with silica gel $60 \mathrm{~F}_{254}$. IR spectra were recorded by using an IR Perkin-Elmer spectrum 1 FTIR spectrophotometer. Proton magnetic resonance spectra were recorded in $\mathrm{CDCl}_{3}$ with Bruker AM 300 spectrometers (RheinstettenForchheim, Germany) operating at $300 \mathrm{MHz}$, respectively. The ${ }^{13} \mathrm{C}$ NMR spectra were recorded in $\mathrm{CDCl}_{3}$ with Bruker AM 100 spectrometer operating at $100 \mathrm{MHz}$. Tetramethyl-silane was used as an internal standard. Elemental analyses for $\mathrm{C}, \mathrm{H}$ and N were recorded with Perkin-Elmer 2400 Series II CHN Analyzer. Melting points were recorded on a GallenKamp apparatus and are uncorrected.

Synthesis of 5-arylfuran-2-carbaldehydes ${ }^{30}: 4.5 \mathrm{~g}$ of substituted aniline was dissolved in a mixture of conc. hydrochloric acid and $20 \mathrm{~mL}$ of water under stirring and cooled in an ice bath at $-5^{\circ} \mathrm{C}$. A solution of sodium nitrite $(2 \mathrm{~g}$ in $10 \mathrm{~mL}$ of water) was added portion wise, keeping the temperature below $7-8{ }^{\circ} \mathrm{C}$. The reaction mixture was left for $1 \mathrm{~h}$ for the completion of diazotization, filtered with the help of glass wool (if there is any turbidity observed). Then to the solution of furan-2-carbaldehyde ( $2 \mathrm{~mL}$ in $10 \mathrm{~mL}$ of acetone and water), the diazonium solution was added drop wise followed by a solution of copper chloride ( $2 \mathrm{~g}$ in $10 \mathrm{~mL}$ of water). The 
TABLE-1

PHYSICAL DATA FOR THE ARYLFURAN-2-CARBALDEHYDES

\begin{tabular}{|c|c|c|c|c|c|c|c|c|}
\hline \multirow{2}{*}{ Compound } & \multirow{2}{*}{$\mathrm{R}$} & \multirow{2}{*}{ Yield (\%) } & \multirow{2}{*}{$\begin{array}{l}\text { m.p. } \\
\left({ }^{\circ} \mathrm{C}\right)\end{array}$} & \multirow{2}{*}{ m.w. } & \multirow{2}{*}{ m.f. } & \multicolumn{3}{|c|}{ Elemental analysis (\%): Calcd. (found) } \\
\hline & & & & & & $\mathrm{C}$ & $\mathrm{H}$ & $\mathrm{N}$ \\
\hline 1 & $2-\mathrm{NO}_{2}$ & 67.00 & $80-81$ & 217 & $\mathrm{C}_{11} \mathrm{H}_{7} \mathrm{NO}_{4}$ & $60.81(60.85)$ & $3.22(3.34)$ & $6.45(6.51)$ \\
\hline 2 & $3-\mathrm{NO}_{2}$ & 61.00 & 148 & 217 & $\mathrm{C}_{11} \mathrm{H}_{7} \mathrm{NO}_{4}$ & $60.81(60.92)$ & $3.22(3.29)$ & $6.45(6.40)$ \\
\hline 3 & $4-\mathrm{NO}_{2}$ & 64.00 & 196 & 217 & $\mathrm{C}_{11} \mathrm{H}_{7} \mathrm{NO}_{4}$ & $60.81(60.86)$ & $3.22(3.34)$ & $6.45(6.52)$ \\
\hline 4 & $2-\mathrm{Cl}$ & 58.00 & 72 & 206,208 & $\mathrm{C}_{11} \mathrm{H}_{7} \mathrm{ClO}_{2}$ & $64.06(63.96)$ & $3.39(3.43)$ & - \\
\hline 5 & $3-\mathrm{Cl}$ & 55.00 & 104 & 206,208 & $\mathrm{C}_{11} \mathrm{H}_{7} \mathrm{ClO}_{2}$ & $64.06(64.12)$ & $3.39(3.45)$ & - \\
\hline 6 & $4-\mathrm{Cl}$ & 62.00 & 118 & 206,208 & $\mathrm{C}_{11} \mathrm{H}_{7} \mathrm{ClO}_{2}$ & $64.06(64.09)$ & $3.39(3.44)$ & - \\
\hline 7 & $4-\mathrm{Br}$ & 61.00 & 150 & 250,252 & $\mathrm{C}_{11} \mathrm{H}_{7} \mathrm{BrO}_{2}$ & $52.62(52.66)$ & $2.80(2.84)$ & - \\
\hline 8 & 4-COOH & 57.00 & 296 & 216 & $\mathrm{C}_{12} \mathrm{H}_{8} \mathrm{O}_{4}$ & $66.66(66.71)$ & $3.70(3.76)$ & - \\
\hline
\end{tabular}

TABLE-2

FTIR AND ${ }^{1}$ H NMR DATA OF ARYLFURAN-2-CARBALDEHYDE

\begin{tabular}{|c|c|c|}
\hline Compound & $\operatorname{IR}\left(\mathrm{cm}^{-1}\right)$ & NMR data $\delta(\mathrm{ppm})$ \\
\hline 1 & $\begin{array}{l}1669.69(\mathrm{C}=\mathrm{O}), 1510.01 \text { and } 1355.83 \\
\left(\text { Asym and sym }-\mathrm{NO}_{2}\right), 2363.67 \text { (aromatic } \\
\text { ring), } 2846.65(\mathrm{C}-\mathrm{H} \text { stretch of aldehyde) }\end{array}$ & $\begin{array}{l}\left.{ }^{1} \mathrm{H} \text { NMR: } 9.671 \text { (s, } 1 \mathrm{H}, \mathrm{CHO}\right), 7.30 \text { (d, } 1 \mathrm{H} \text {, furyl proton), } 6.780 \text { (d, } 1 \mathrm{H} \text {, furyl proton), } \\
7.836-7.531(\mathrm{~m}, 4 \mathrm{H}, \mathrm{Ar}-\mathrm{H},) ;{ }^{13} \mathrm{C} \text { NMR: } 177.72(\mathrm{C}=\mathrm{O} \text { aldehyde }), 153.37,152.93, \\
132.41,130.31,130.07,126.65,124.32,122.99,121.83,112.03 \text { (Ar-C) }\end{array}$ \\
\hline 2 & $\begin{array}{l}1678.69(\mathrm{C}=\mathrm{O}), 1504.32 \text { and } 1359.64 \\
\left(\text { Asym and sym }-\mathrm{NO}_{2}\right), 2361.51 \text { (aromatic } \\
\text { ring) }\end{array}$ & $\begin{array}{l}{ }^{1} \mathrm{H} \text { NMR: } 9.709(\mathrm{~s}, 1 \mathrm{H}, \mathrm{Ar}-\mathrm{CHO}), 7.350(\mathrm{~d}, 1 \mathrm{H} \text {, furyl proton }), 6.994(\mathrm{~d}, 1 \mathrm{H}, \text { furyl } \\
\text { proton }), 8.614(\mathrm{~s}, 1 \mathrm{H}, \mathrm{Ar}-\mathrm{H}), 8.232(\mathrm{~d}, 1 \mathrm{H}, \mathrm{Ar}-\mathrm{H}), 8.142(\mathrm{~d}, 1 \mathrm{H}, \mathrm{Ar}-\mathrm{H}), 7.655(\mathrm{t}, 1 \mathrm{H} \text {, } \\
\text { Ar-H); }{ }^{13} \mathrm{C} \text { NMR: } 177.49(\mathrm{C}=\mathrm{O} \text { aldehyde }), 157.81,152.74,146.67,133.94,132.78, \\
130.62,130.20,128.82,120.06,109.99(\mathrm{Ar}-\mathrm{C})\end{array}$ \\
\hline 4 & $\begin{array}{l}1670.01(\mathrm{C}=\mathrm{O}), 2359.12 \text { (Aromatic ring), } \\
1089.91(\mathrm{C}-\mathrm{Cl})\end{array}$ & $\begin{array}{l}{ }^{1} \mathrm{H} \text { NMR: } 9.659(\mathrm{~s}, 1 \mathrm{H}, \mathrm{Ar}-\mathrm{CHO}), 7.308(\mathrm{~d}, 1 \mathrm{H}, \text { furyl proton }), 6.850(\mathrm{~d}, 1 \mathrm{H}, \text { furyl } \\
\text { proton }), 7.796(\mathrm{~d}, 1 \mathrm{H}, \mathrm{Ar}-\mathrm{H}), 7.679(\mathrm{t}, 1 \mathrm{H}, \mathrm{Ar}-\mathrm{H}), 7.369(\mathrm{t}, 2 \mathrm{H}, \mathrm{Ar}-\mathrm{H}) ;{ }^{13} \mathrm{C} \text { NMR: } \\
177.49(\mathrm{C}=\mathrm{O} \text { aldehyde }), 155.43,151.56,131.55,130.95,130.17,129.15,127.62 \text {, } \\
127.17,122.87,113.19 \text { (Ar-C) }\end{array}$ \\
\hline 5 & $\begin{array}{l}1669.52(\mathrm{C}=\mathrm{O}) 2360.04 \text { (Aromatic ring), } \\
2833.99(\mathrm{C}-\mathrm{H} \text { stretch of aldehydes), } \\
1097.98(\mathrm{C}-\mathrm{Cl})\end{array}$ & $\begin{array}{l}\left.{ }^{1} \mathrm{H} \text { NMR: } 9.681 \text { (s, } 1 \mathrm{H}, \text { Ar-CHO }\right), 7.460(\mathrm{~d}, 1 \mathrm{H}, \text { furyl proton }), 7.994(\mathrm{~d}, 1 \mathrm{H}, \mathrm{Ar}-\mathrm{H}) \\
7.299(\mathrm{~d}, 1 \mathrm{H}, \text { furyl proton }), 7.383(\mathrm{~m}, 2 \mathrm{H}, \mathrm{Ar}-\mathrm{H}), 7.235(\mathrm{~d}, 1 \mathrm{H}, \mathrm{Ar}-\mathrm{H}) ;{ }^{13} \mathrm{C} \text { NMR: } \\
177.35(\mathrm{C}=\mathrm{O} \text { aldehyde }), 152.35,135.16,130.67,130.28,129.61,125.28,123.34 \\
123.06,112.65,108.50 \text { (Ar-C) }\end{array}$ \\
\hline 6 & $\begin{array}{l}1661.78(\mathrm{C}=\mathrm{O}), 2360.11 \text { (aromatic ring), } \\
1094.28(\mathrm{C}-\mathrm{Cl})\end{array}$ & $\begin{array}{l}{ }^{1} \mathrm{H} \text { NMR: } 9.639(\mathrm{~s}, 1 \mathrm{H}, \mathrm{Ar}-\mathrm{CHO}), 7.293(\mathrm{~d}, 1 \mathrm{H}, \text { furyl proton }), 6.808(\mathrm{~d}, 1 \mathrm{H}, \text { furyl } \\
\text { proton }), 7.750(\mathrm{~d}, 2 \mathrm{H}, \mathrm{Ar}-\mathrm{H},) 7.417(\mathrm{~d}, 2 \mathrm{H}, \mathrm{Ar}-\mathrm{H}) ;{ }^{13} \mathrm{C} \text { NMR: } 177.21(\mathrm{C}=\mathrm{O} \text { aldehyde), } \\
\text { 158.20, } 152.14,135.66,129.26,127.45,126.50,123.41,107.96(\mathrm{Ar}-\mathrm{C})\end{array}$ \\
\hline 7 & $\begin{array}{l}1661.73(\mathrm{C}=\mathrm{O}), 2863.39(\mathrm{C}-\mathrm{H} \text { stretch of } \\
\text { aldehyde), } 2360.38 \text { (aromatic ring), } \\
1041.23(\mathrm{C}-\mathrm{Br} \text { bond) }\end{array}$ & $\begin{array}{l}{ }^{1} \mathrm{H} \text { NMR: } 9.641(\mathrm{~s}, 1 \mathrm{H}, \mathrm{Ar}-\mathrm{CHO}), 7.240(\mathrm{~d}, 1 \mathrm{H}, \text { furyl proton }), 6.829(\mathrm{~d}, 1 \mathrm{H}, \text { furyl } \\
\text { proton }), 7.682-7.290(\mathrm{~m}, 4 \mathrm{H}, \mathrm{Ar}-\mathrm{H}) ;{ }^{13} \mathrm{C} \text { NMR: } 177.25(\mathrm{C}=\mathrm{O} \text { aldehyde }), 132.24, \\
127.92,126.72,123.96,123.69,123.33,108.06,108.01(\mathrm{Ar}-\mathrm{C})\end{array}$ \\
\hline 8 & $\begin{array}{l}2360.14 \text { (aromatic ring), } 1672.39(\mathrm{C}=\mathrm{O} \\
\text { acid), } 2968.44(-\mathrm{OH} \text { acid) } 1610.63(\mathrm{C}=\mathrm{O} \\
\text { aldehyde) }\end{array}$ & $\begin{array}{l}{ }^{1} \mathrm{H} \text { NMR: } 9.567(\mathrm{~s}, 1 \mathrm{H}, \mathrm{Ar}-\mathrm{CHO}), 7.797(\mathrm{~d}, 1 \mathrm{H}, \mathrm{Ar}-\mathrm{H}), 7.877(\mathrm{~d}, 1 \mathrm{H}, \mathrm{Ar}-\mathrm{H}), 7.288(\mathrm{~d} \text {, } \\
1 \mathrm{H}, \mathrm{Ar}-\mathrm{H}), 8.021(\mathrm{~d}, 1 \mathrm{H}, \mathrm{Ar}-\mathrm{H}), 6.893(\mathrm{~d}, 1 \mathrm{H} \text {, furyl proton }), 7.238(\mathrm{~s}, 1 \mathrm{H}, \text { furyl proton }) \\
{ }^{13} \mathrm{C} \text { NMR: } 178.51(\mathrm{C}=\mathrm{O} \text { aldehyde }), 165.96(\mathrm{C}=\mathrm{O} \text { of COOH}) 155.43,153.90,132.46 \text {, } \\
\text { 127.65, 123.87, 122.43, 122.09, } 117.78,109.89(\mathrm{Ar}-\mathrm{C})\end{array}$ \\
\hline
\end{tabular}

temperature was raised to $30^{\circ} \mathrm{C}$ by heating (if necessary) and stirred for 4-6 h then left for $24 \mathrm{~h}$ at room temperature. Precipitates obtained were filtered, dried and recrystallized from ethanol. Furan-2-carbaldehydes thus prepared are listed in Table-1 and their spectral data in Table-2.

Synthesis of chalcones: Equimolar quantities of an arylfuran-2-carbaldehyde and appropriate acetophenone was taken in ethanol and water mixture $(10 \mathrm{~mL}$ ethanol $+10 \mathrm{~mL}$ water) in the presence of $6 \mathrm{~g}$ of $\mathrm{NaOH}$ as a catalyst in ice bath $\left(-5^{\circ} \mathrm{C}\right)$ and the mixture was stirred for $4 \mathrm{~h}$. Solid product formed was filtered, dried and recrystallized from ethanol. The chalcones thus prepared are listed in the Table- 3 and their spectral data in the Table-4 (Scheme-I).

RESULTS AND DISCUSSION

A convenient method for the synthesis of arylfurfural is based on the catalytic arylation of furfural with arenediazonium salts in 40-70\% yield which is fairly good for this reaction. The best yields in the arylation of furfural were obtained with diazonium salts containing a nitro group or two halogen atoms in the aromatic ring. In the present work, we synthesized different chalcones of arylfurfural with various acetophenones using basic catalyst $(\mathrm{NaOH})$ according to Claisen-Schmidt condensation.
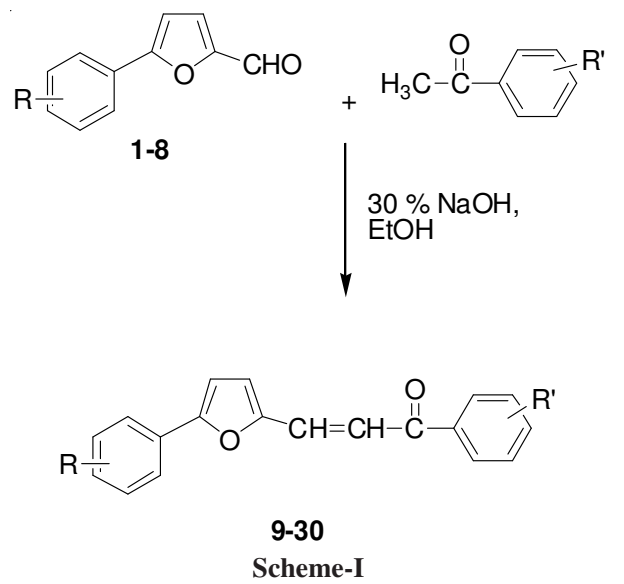
TABLE-3

PHYSICAL DATA FOR THE CHALCONES

\begin{tabular}{|c|c|c|c|c|c|c|c|c|c|}
\hline \multirow{2}{*}{ Compd. } & \multirow{2}{*}{$\begin{array}{c}\text { Yield } \\
(\%)\end{array}$} & \multirow{2}{*}{$\begin{array}{l}\text { m.p. } \\
\left({ }^{\circ} \mathrm{C}\right)\end{array}$} & \multirow{2}{*}{ m.w. } & \multirow{2}{*}{ m.f. } & \multicolumn{3}{|c|}{ Elemental analysis (\%): Calcd. (found) } & \multirow[b]{2}{*}{$\mathrm{R}$} & \multirow[b]{2}{*}{$\mathrm{R}^{\prime}$} \\
\hline & & & & & $\mathrm{C}$ & $\mathrm{H}$ & $\mathrm{N}$ & & \\
\hline 9 & 71 & $172 *$ & 319 & $\mathrm{C}_{19} \mathrm{H}_{13} \mathrm{NO}_{4}$ & $71.47(71.27)$ & $4.07(3.90)$ & $4.38(4.36)$ & $4-\mathrm{NO}_{2}$ & $\mathrm{H}$ \\
\hline 10 & 68 & 126 & 308,310 & $\mathrm{C}_{19} \mathrm{H}_{13} \mathrm{ClO}_{2}$ & $74.02(73.90)$ & $4.22(4.18)$ & - & $4-\mathrm{Cl}$ & $\mathrm{H}$ \\
\hline 11 & 77 & 140 & 352,354 & $\mathrm{C}_{19} \mathrm{H}_{13} \mathrm{BrO}_{2}$ & $64.77(64.91)$ & $3.69(3.44)$ & - & $4-\mathrm{Br}$ & $\mathrm{H}$ \\
\hline 12 & 72 & 136 & 319 & $\mathrm{C}_{19} \mathrm{H}_{13} \mathrm{NO}_{4}$ & $71.47(71.43)$ & $4.07(4.01)$ & $4.38(4.20)$ & $3-\mathrm{NO}_{2}$ & $\mathrm{H}$ \\
\hline 13 & 69 & $200 d$ & 318 & $\mathrm{C}_{20} \mathrm{H}_{14} \mathrm{O}_{4}$ & $75.47(75.34)$ & $4.40(4.24)$ & - & 4-COOH & $\mathrm{H}$ \\
\hline 14 & 80 & 238 & 397, 399 & $\mathrm{C}_{19} \mathrm{H}_{12} \mathrm{BrNO}_{4}$ & $57.43(57.47)$ & $3.02(2.98)$ & $3.52(3.59)$ & $4-\mathrm{Br}$ & $4-\mathrm{NO}_{2}$ \\
\hline 15 & 73 & $192 \mathrm{~d}$ & 364 & $\mathrm{C}_{19} \mathrm{H}_{12} \mathrm{~N}_{2} \mathrm{O}_{6}$ & $62.63(62.60)$ & $3.29(3.15)$ & $7.69(7.63)$ & $3-\mathrm{NO}_{2}$ & $4-\mathrm{NO}_{2}$ \\
\hline 16 & 72 & $200 \mathrm{~d}$ & 364 & $\mathrm{C}_{19} \mathrm{H}_{12} \mathrm{~N}_{2} \mathrm{O}_{6}$ & $62.63(62.65)$ & $3.29(3.33)$ & $7.69(7.65)$ & $2-\mathrm{NO}_{2}$ & $4-\mathrm{NO}_{2}$ \\
\hline 17 & 65 & 188 & 353 & $\mathrm{C}_{19} \mathrm{H}_{12} \mathrm{ClNO}_{4}$ & $64.58(64.55)$ & $3.39(3.32)$ & $3.96(3.91)$ & $3-\mathrm{Cl}$ & $4-\mathrm{NO}_{2}$ \\
\hline 18 & 70 & $150 \mathrm{~d}$ & 353,355 & $\mathrm{C}_{19} \mathrm{H}_{12} \mathrm{ClNO}_{4}$ & $64.58(64.49)$ & $3.39(3.34)$ & $3.96(3.92)$ & $4-\mathrm{Cl}$ & $4-\mathrm{NO}_{2}$ \\
\hline 19 & 76 & $228 \mathrm{~d}$ & 364 & $\mathrm{C}_{19} \mathrm{H}_{12} \mathrm{~N}_{2} \mathrm{O}_{6}$ & $62.63(62.56)$ & $3.29(3.36)$ & $7.69(7.72)$ & $4-\mathrm{NO}_{2}$ & $4-\mathrm{NO}_{2}$ \\
\hline 20 & 68 & $140 \mathrm{~d}$ & 353,355 & $\mathrm{C}_{19} \mathrm{H}_{12} \mathrm{ClNO}_{4}$ & $64.58(64.50)$ & $3.39(3.28)$ & $3.96(3.92)$ & $2-\mathrm{Cl}$ & $4-\mathrm{NO}_{2}$ \\
\hline 21 & 65 & $134-36$ & 342 & $\mathrm{C}_{19} \mathrm{H}_{12} \mathrm{Cl}_{2} \mathrm{O}_{2}$ & $66.66(66.71)$ & $3.50(3.46)$ & - & $4-\mathrm{Cl}$ & $4-\mathrm{Cl}$ \\
\hline 23 & 63 & $144-46$ & - & $\mathrm{C}_{19} \mathrm{H}_{11} \mathrm{BrClO}_{2}$ & $59.06(58.95)$ & $3.10(2.95)$ & - & $4-\mathrm{Br}$ & $4-\mathrm{Cl}$ \\
\hline 24 & 62 & 118 & - & $\mathrm{C}_{19} \mathrm{H}_{12} \mathrm{Cl}_{2} \mathrm{O}_{2}$ & $66.66(66.75)$ & $3.50(3.37)$ & - & $2-\mathrm{Cl}$ & $4-\mathrm{Cl}$ \\
\hline 25 & 67 & 98 & - & $\mathrm{C}_{19} \mathrm{H}_{12} \mathrm{Cl}_{2} \mathrm{O}_{2}$ & $66.66(66.60)$ & $3.50(3.43)$ & - & $3-\mathrm{Cl}$ & $4-\mathrm{Cl}$ \\
\hline 26 & 73 & $200 d$ & 352,354 & $\mathrm{C}_{20} \mathrm{H}_{13} \mathrm{ClO}_{4}$ & $68.18(68.13)$ & $3.69(3.61)$ & - & 4-COOH & $4-\mathrm{Cl}$ \\
\hline 27 & 75 & 160 & 397,399 & $\mathrm{C}_{19} \mathrm{H}_{12} \mathrm{BrNO}_{4}$ & $57.43(57.28)$ & 3.02 (2.97) & $3.52(3.42)$ & $3-\mathrm{NO}_{2}$ & $4-\mathrm{Br}$ \\
\hline 28 & 71 & 158 & - & $\mathrm{C}_{19} \mathrm{H}_{12} \mathrm{Br}_{2} \mathrm{O}_{2}$ & $52.90(52.95)$ & $2.79(2.83)$ & - & $4-\mathrm{Br}$ & $4-\mathrm{Br}$ \\
\hline 29 & 81 & 146 & 353,355 & $\mathrm{C}_{19} \mathrm{H}_{12} \mathrm{CINO}_{4}$ & $64.58(64.61)$ & $3.39(3.32)$ & $3.96(3.89)$ & $4-\mathrm{Cl}$ & $3-\mathrm{NO}_{2}$ \\
\hline 30 & 76 & 148 & 364 & $\mathrm{C}_{19} \mathrm{H}_{12} \mathrm{~N}_{2} \mathrm{O}_{6}$ & $62.63(62.69)$ & $3.23(3.29)$ & $7.69(7.62)$ & $2-\mathrm{NO}_{2}$ & $3-\mathrm{NO}_{2}$ \\
\hline
\end{tabular}

TABLE-4

FTIR AND ${ }^{1} \mathrm{H}$ NMR DATA OF CHALCONE

\begin{tabular}{|c|c|c|}
\hline Compound & $\operatorname{IR}\left(\mathrm{cm}^{-1}\right)$ & NMR data $\delta(p p m)$ \\
\hline 9 & $\begin{array}{l}2341.12 \text { (Aromatic ring) } 1664.91(\mathrm{C}=\mathrm{O} \\
\text { conjugated carbonyl group), } 1593.85(\mathrm{C}=\mathrm{C} \\
\text { conjugated) } 1562.44 \text { and } 1326.24(\text { Asym } \\
\left.\text { and sym }-\mathrm{NO}_{2}\right) \text {. }\end{array}$ & $\begin{array}{l}{ }^{1} \mathrm{H} \text { NMR: } 8.539(\mathrm{~d}, 1 \mathrm{H}, \mathrm{Ar}-\mathrm{H}), 7.701(\mathrm{~d}, 1 \mathrm{H}, \mathrm{Ar}-\mathrm{H}), 7.610(\mathrm{~d}, 1 \mathrm{H}, \mathrm{Ar}-\mathrm{H}), 7.572(\mathrm{~d}, 1 \mathrm{H} \text {, } \\
\text { Ar-H), } 7.523(\mathrm{~d}, 1 \mathrm{H}, \mathrm{Ar}-\mathrm{H}), 7.486(\mathrm{~d}, 1 \mathrm{H}, \mathrm{Ar}-\mathrm{H}), 7.404(\mathrm{~d}, 1 \mathrm{H}, \text { ethylenic }), 7.220(\mathrm{~d}, \\
1 \mathrm{H}, \text { ethylenic }), 6.801(\mathrm{~d}, 1 \mathrm{H}, \text { furyl proton }), 6.764(\mathrm{~d}, 1 \mathrm{H}, \text { furyl proton }),{ }^{13} \mathrm{C} \text { NMR: } \\
189.56(\mathrm{C}=\mathrm{O}), 132.99,128.48(\mathrm{C}=\mathrm{C}), 153.58,152.96,142.35,141.46,138.05,135.36 \text {, } \\
129.90,129.28128 .72,124.46,120.59,118.37,111.63 \text { (Ar-C). }\end{array}$ \\
\hline 10 & $\begin{array}{l}2363.83 \text { (Aromatic ring) } 1646.14 \mathrm{~cm}^{-1} \\
(\mathrm{C}=\mathrm{O} \text { conjugated carbonyl group), } 1585.50 \\
\mathrm{cm}^{-1}\left(\mathrm{C}=\mathrm{C} \text { conjugated) } 1572.71 \mathrm{~cm}^{-1} \text { and }\right. \\
\left.1338.06 \text { (Asym and sym }-\mathrm{NO}_{2}\right), 1092.68 \\
\mathrm{~cm}^{-1}(\mathrm{C}-\mathrm{Cl} \text { bond). }\end{array}$ & $\begin{array}{l}{ }^{1} \mathrm{H} \text { NMR: } 8.048(\mathrm{~d}, 1 \mathrm{H}, \mathrm{Ar}-\mathrm{H}), 7.701(\mathrm{~d}, 1 \mathrm{H}, \mathrm{Ar}-\mathrm{H}), 7.610(\mathrm{~d}, 1 \mathrm{H}, \mathrm{Ar}-\mathrm{H}), 7.572(\mathrm{~d}, 1 \mathrm{H}, \\
\text { Ar-H), } 7.523(\mathrm{~d}, 1 \mathrm{H}, \mathrm{Ar}-\mathrm{H}), 7.486(\mathrm{~d}, 1 \mathrm{H}, \mathrm{Ar}-\mathrm{H}), 7.404(\mathrm{~d}, 1 \mathrm{H}, \text { ethylenic }), 7.458(\mathrm{~d}, \\
1 \mathrm{H}, \text { ethylenic }), 6.801(\mathrm{~d}, 1 \mathrm{H}, \text { furyl proton }), 6.764(\mathrm{~d}, 1 \mathrm{H}, \text { furyl proton }),{ }^{13} \mathrm{C} \mathrm{NMR}: \\
189.78(\mathrm{C}=\mathrm{O}) 130.34,129.15,(\mathrm{C}=\mathrm{C}), 155.24,151.43138 .30,134.40,132.74,128.63, \\
128.43,125.69,119.12,118.69,108.59(\mathrm{Ar}-\mathrm{C}) .\end{array}$ \\
\hline 11 & $\begin{array}{l}2981.98 \mathrm{~cm}^{-1}(-\mathrm{OH}), 1651.68 \mathrm{~cm}^{-1}(\mathrm{C}=\mathrm{O}) \\
2359.79 \mathrm{~cm}^{-1} \text { (Aromatic ring), } 1031.12 \mathrm{~cm}^{-1} \\
\text { (C-Br bond). }\end{array}$ & $\begin{array}{l}{ }^{1} \mathrm{H} \text { NMR: } 8.047(\mathrm{~d}, 1 \mathrm{H}, \mathrm{Ar}-\mathrm{H}), 7.735-7.486(\mathrm{~m}, 8 \mathrm{H}, \mathrm{Ar}-\mathrm{H}), 7.474(\mathrm{~d}, 1 \mathrm{H}, \text { ethylenic }), \\
7.461(\mathrm{~d}, 1 \mathrm{H} \text {, ethylenic), } 6.799(\mathrm{~d}, 1 \mathrm{H} \text {, furyl proton }), 6.776(\mathrm{~d}, 1 \mathrm{H}, \text { furyl proton); } \\
{ }^{13} \mathrm{C} \text { NMR: } 189.77(\mathrm{C}=\mathrm{O}), 130.34,128.71(\mathrm{C}=\mathrm{C}) 155.23,151.43,138.24,132.77, \\
\text { 132.07, } 128.63,128.43,125.89,122.56,119.09,118.77,108.70 \text { (Ar-C). }\end{array}$ \\
\hline 12 & $\begin{array}{l}2364.56 \mathrm{~cm}^{-1} \text { (Aromatic ring) } 1659.63 \mathrm{~cm}^{-1} \\
(\mathrm{C}=\mathrm{O} \text { conjugated carbonyl group), } 1593.47 \\
\mathrm{cm}^{-1}(\mathrm{C}=\mathrm{C} \text { conjugated }), 1562.76 \mathrm{~cm}^{-1} \text { and } \\
\left.1350.11 \text { (Asym and sym- } \mathrm{NO}_{2}\right) .\end{array}$ & $\begin{array}{l}{ }^{1} \mathrm{H} \text { NMR: } 8.578(\mathrm{~s}, 1 \mathrm{H}, \mathrm{Ar}-\mathrm{H},), 7.636-7.505(\mathrm{~m}, 8 \mathrm{H}, \mathrm{Ar}-\mathrm{H}), 8.176(\mathrm{~d}, 1 \mathrm{H} \text {, ethylenic }), \\
6.934(\mathrm{~d}, 1 \mathrm{H} \text {, furyl proton }), 6.845(\mathrm{~d}, 1 \mathrm{H}, \text { furyl proton }), 8.050(\mathrm{~d}, 1 \mathrm{H} \text {, ethylenic }) ;{ }^{13} \mathrm{C} \\
\text { NMR: } 189.64(\mathrm{C}=\mathrm{O}), 130.00,128.49,(\mathrm{C}=\mathrm{C}), 153.50,152.30148 .86,138.06,132.92 \text {, } \\
\text { 131.42, } 129.97,129.81,128.70,122.78,120.07,119.13,118.37,110.20 \text { (Ar-C). }\end{array}$ \\
\hline 13 & $\begin{array}{l}2361.30 \mathrm{~cm}^{-1} \text { (Aromatic ring) } 1687.97 \mathrm{~cm}^{-1} \\
(\mathrm{C}=\mathrm{O} \text { acid }), 1573.97 \mathrm{~cm}^{-1}(\mathrm{C}=\mathrm{C} \\
\text { conjugated }), 1532.37 \mathrm{~cm}^{-1}(\mathrm{C}=\mathrm{O} \text { aldehyde })\end{array}$ & $\begin{array}{l}{ }^{1} \mathrm{H} \text { NMR: } 8.048(\mathrm{~d}, 1 \mathrm{H}, \mathrm{Ar}-\mathrm{H}), 7.745-7.480(\mathrm{~m}, 8 \mathrm{H}, \mathrm{Ar}-\mathrm{H}), 7.470(\mathrm{~d}, 1 \mathrm{H}, \text { ethylenic }), \\
7.345(\mathrm{~d}, 1 \mathrm{H}, \text { ethylenic }), 6.80(\mathrm{~d}, 1 \mathrm{H}, \text { furyl proton }), 6.764(\mathrm{~d}, 1 \mathrm{H} \text {, furyl proton }) \\
{ }^{13} \mathrm{C} \text { NMR: } 189.6481(\mathrm{C}=\mathrm{O}), 131.89,129.42(\mathrm{C}=\mathrm{C}), 168.74(\mathrm{C}=\mathrm{O} \text { of acid }), 151.72, \\
151.09,134.40,134.14,130.97,130.68,129.87,129.62,124.88,123.59,110.32, \\
107.59 \text { (Ar-C). }\end{array}$ \\
\hline 14 & $\begin{array}{l}2340.06 \mathrm{~cm}^{-1} \text { (Aromatic ring) } 1658.29 \mathrm{~cm}^{-1} \\
(\mathrm{C}=\mathrm{O} \text { conjugated carbonyl group), } 1584.37 \\
\mathrm{cm}^{-1}(\mathrm{C}=\mathrm{C} \text { conjugated }), 1342.49 \text { (sym - } \\
\left.\mathrm{NO}_{2}\right), 1022.50 \mathrm{~cm}^{-1}(\mathrm{C}-\mathrm{Br} \text { bond })\end{array}$ & $\begin{array}{l}{ }^{1} \mathrm{H} \text { NMR: } 8.358(\mathrm{~d}, 2 \mathrm{H}, \mathrm{Ar}-\mathrm{H}), 8.173(\mathrm{~d}, 2 \mathrm{H}, \mathrm{Ar}-\mathrm{H}), 7.643(\mathrm{~d}, 1 \mathrm{H}, \mathrm{Ar}-\mathrm{H}), 7.572(\mathrm{~d}, 1 \mathrm{H} \text {, } \\
\text { Ar-H), } 7.448(\mathrm{~d}, 1 \mathrm{H}, \mathrm{Ar}-\mathrm{H}), 7.410(\mathrm{~d}, 1 \mathrm{H} \text {, ethylenic }), 7.315(\mathrm{~d}, 1 \mathrm{H}, \text { ethylenic }), 6.877 \\
(\mathrm{~d}, 1 \mathrm{H}, \text { furyl proton }), 6.808(\mathrm{~d}, 1 \mathrm{H} \text {, furyl proton }),{ }^{13} \mathrm{C} \text { NMR: } 187.63(\mathrm{C}=\mathrm{O}), 131.69, \\
128.30(\mathrm{C}=\mathrm{C}), 155.01,150.90,149.74,142.39,131.95,131.15,126.35,123.83, \\
122.00,120.88,118.47,110.14 \text { (Ar-C). }\end{array}$ \\
\hline 15 & $\begin{array}{l}2360.43 \mathrm{~cm}^{-1} \text { (Aromatic ring) } 1663.30 \mathrm{~cm}^{-1} \\
(\mathrm{C}=\mathrm{O} \text { conjugated carbonyl group), } 1588.99 \\
\mathrm{cm}^{-1}(\mathrm{C}=\mathrm{C} \text { conjugated }), 1560.33 \mathrm{~cm}^{-1} \text { and } \\
\left.1350.49 \text { (Asym and sym }-\mathrm{NO}_{2}\right)\end{array}$ & $\begin{array}{l}{ }^{1} \mathrm{H} \text { NMR: } 8.619(\mathrm{~d}, 2 \mathrm{H}, \mathrm{Ar}-\mathrm{H}), 8.379(\mathrm{~d}, 2 \mathrm{H}, \mathrm{Ar}-\mathrm{H}), 8.203(\mathrm{~d}, 1 \mathrm{H}, \mathrm{Ar}-\mathrm{H}), 8.071(\mathrm{~d}, 1 \mathrm{H}, \\
\text { Ar-H), } 7.675(\mathrm{~d}, 1 \mathrm{H}, \mathrm{Ar}-\mathrm{H}), 7.520(\mathrm{~d}, 1 \mathrm{H}, \mathrm{Ar}-\mathrm{H}), 7.353(\mathrm{~d}, 1 \mathrm{H}, \text { ethylenic }), 7.324(\mathrm{~d}, \\
1 \mathrm{H}, \text { ethylenic }), 6.996(\mathrm{~d}, 1 \mathrm{H}, \text { furyl proton }), 6.923(\mathrm{~d}, 1 \mathrm{H}, \text { furyl proton }),{ }^{13} \mathrm{C} \text { NMR: } \\
178.36(\mathrm{C}=\mathrm{O}), 131.01,124.87(\mathrm{C}=\mathrm{C}), 155.47,152.23,148.57,130.93,130.70,130.42 \text {, } \\
129.75,123.90,123.06,122.35,120.62,119.21,118.66,111.67,110.83(\text { Ar-C). }\end{array}$ \\
\hline 16 & $\begin{array}{l}1661.33 \mathrm{~cm}^{-1}(\mathrm{C}=\mathrm{O} \text { conjugated carbonyl } \\
\text { group }), 1587.85 \mathrm{~cm}^{-1}(\mathrm{C}=\mathrm{C} \text { conjugated }) \\
1576.38 \mathrm{~cm}^{-1} \text { and } 1344.81 \text { (Asym and sym } \\
\left.-\mathrm{NO}_{2}\right) .\end{array}$ & $\begin{array}{l}{ }^{1} \mathrm{H} \text { NMR: } 7.773(\mathrm{~d}, 1 \mathrm{H}, \text { Ar-H}), 7.650(\mathrm{~d}, 1 \mathrm{H}, \mathrm{Ar}-\mathrm{H}), 7.578(\mathrm{~d}, 1 \mathrm{H}, \mathrm{Ar}-\mathrm{H}), 7.525(\mathrm{~d}, 1 \mathrm{H} \text {, } \\
\text { Ar-H), } 7.431(\mathrm{~d}, 1 \mathrm{H} \text {, ethylenic }), 7.395(\mathrm{~d}, 1 \mathrm{H} \text {, ethylenic }), 6.861(\mathrm{~d}, 1 \mathrm{H}, \text { furyl proton }), \\
6.809(\mathrm{~d}, 1 \mathrm{H} \text {, furyl proton }) ;{ }^{13} \mathrm{C} \text { NMR: } 178.24(\mathrm{C}=\mathrm{O}), 130.23,124.42(\mathrm{C}=\mathrm{C}), 154.90, \\
153.17,147.14,133.02,132.51,129.73,129.08,128.18,123.95,123.58,122.99, \\
122.32,119.75,112.94,112.30 \text { (Ar-C). }\end{array}$ \\
\hline
\end{tabular}




\begin{tabular}{|c|c|c|}
\hline Compound & $\operatorname{IR}\left(\mathrm{cm}^{-1}\right)$ & NMR data $\delta(\mathrm{ppm})$ \\
\hline 17 & $\begin{array}{l}1656.05 \mathrm{~cm}^{-1}(\mathrm{C}=\mathrm{O} \text { conjugated carbonyl } \\
\text { group }), 1587.51 \mathrm{~cm}^{-1}(\mathrm{C}=\mathrm{C} \text { conjugated }) \\
1568.26 \mathrm{~cm}^{-1} \text { and } 1343.40 \text { (Asym and sym } \\
\left.-\mathrm{NO}_{2}\right), 1022.88 \mathrm{~cm}^{-1}(\mathrm{C}-\mathrm{Cl} \text { bond). }\end{array}$ & $\begin{array}{l}{ }^{1} \mathrm{H} \text { NMR: } 8.371(\mathrm{~s}, 1 \mathrm{H}, \mathrm{Ar}-\mathrm{H},), 8.199(\mathrm{~d}, 1 \mathrm{H}, \mathrm{Ar}-\mathrm{H}), 8.168(\mathrm{~d}, 1 \mathrm{H}, \mathrm{Ar}-\mathrm{H}), 7.659(\mathrm{~d}, \\
1 \mathrm{H}, \mathrm{Ar}-\mathrm{H}), 7.627(\mathrm{~d}, 1 \mathrm{H}, \text { Ar-H), } 7.476(\mathrm{~d}, 1 \mathrm{H}, \mathrm{Ar}-\mathrm{H}), 7.396(\mathrm{~d}, 1 \mathrm{H}, \mathrm{Ar}-\mathrm{H}), 7.335(\mathrm{~d}, \\
1 \mathrm{H}, \text { Ar-H), } 7.302(\mathrm{~d}, 1 \mathrm{H}, \text { ethylenic }), 7.240(\mathrm{~d}, 1 \mathrm{H}, \text { ethylenic }), 6.884(\mathrm{~d}, 1 \mathrm{H}, \text { furyl } \\
\text { proton }), 6.828(\mathrm{~d}, 1 \mathrm{H}, \text { furyl proton }) ;{ }^{13} \mathrm{C} \text { NMR: } 187.67(\mathrm{C}=\mathrm{O}), 131.08,128.48(\mathrm{C}=\mathrm{C}), \\
154.44,151.11,149.74,142.33,133.95,131.10,130.86,129.73,123.89,123.82, \\
123.00,120.71,118.77,110.69 \text { (Ar-C). }\end{array}$ \\
\hline 18 & $\begin{array}{l}2339.23 \mathrm{~cm}^{-1} \text { (Aromatic ring) } 1656.80 \mathrm{~cm}^{-1} \\
(\mathrm{C}=\mathrm{O} \text { conjugated carbonyl group), } \\
1585.20 \mathrm{~cm}^{-1}(\mathrm{C}=\mathrm{C} \text { conjugated }), 1549.97 \\
\left.\mathrm{~cm}^{-1} \text { and } 1341.45 \text { (Asym and sym }-\mathrm{NO}_{2}\right) \\
1021.94 \mathrm{~cm}^{-1} \text { (C-Cl bond). }\end{array}$ & $\begin{array}{l}{ }^{1} \mathrm{H} \text { NMR: } 8.360(\mathrm{~d}, 2 \mathrm{H}, \mathrm{Ar}-\mathrm{H}), 8.175(\mathrm{~d}, 2 \mathrm{H}, \mathrm{Ar}-\mathrm{H}), 7.711(\mathrm{~d}, 4 \mathrm{H}, \mathrm{Ar}-\mathrm{H}), 7.449(\mathrm{~d}, 1 \mathrm{H} \text {, } \\
\text { ethylenic), } 7.406(\mathrm{~d}, 1 \mathrm{H} \text {, ethylenic }), 6.881(\mathrm{~d}, 1 \mathrm{H} \text {, furyl proton }), 6.796(\mathrm{~d}, 1 \mathrm{H}, \text { furyl } \\
\text { proton }) ;{ }^{13} \mathrm{C} \text { NMR: } 187.61(\mathrm{C}=\mathrm{O}), 131.20,126.20(\mathrm{C}=\mathrm{C}), 155.00,150.91,149.77 \text {, } \\
142.40,133.36,129.75,129.11,128.97,128.01,125.22,123.89,121.00,118.40 \text {, } \\
110.15(\text { Ar-C). }\end{array}$ \\
\hline 19 & $\begin{array}{l}2359.78 \mathrm{~cm}^{-1} \text { (Aromatic ring) } 1661.97 \mathrm{~cm}^{-1} \\
(\mathrm{C}=\mathrm{O} \text { conjugated carbonyl group), } \\
1588.19 \mathrm{~cm}^{-1}(\mathrm{C}=\mathrm{C} \text { conjugated }), 1557.96 \\
\left.\mathrm{~cm}^{-1} \text { and } 1333.57 \text { (Asym and sym }-\mathrm{NO}_{2}\right)\end{array}$ & $\begin{array}{l}{ }^{1} \mathrm{H} \text { NMR: } 8.380-7.630(\mathrm{~m}, 8 \mathrm{H}, \mathrm{Ar}-\mathrm{H}), 7.524(\mathrm{~d}, 1 \mathrm{H} \text {, ethylenic }), 7.325(\mathrm{~d}, 1 \mathrm{H}, \\
\text { ethylenic }), 7.015(\mathrm{~d}, 1 \mathrm{H} \text {, furyl proton }), 6.936(\mathrm{~d}, 1 \mathrm{H}, \text { furyl proton }){ }^{13} \mathrm{C} \text { NMR: } 187.77 \\
(\mathrm{C}=\mathrm{O}), 130.95,125.19(\mathrm{C}=\mathrm{C}), 153.64,152.35,149.84,146.75,142.25,134.85 \\
129.79,124.40,123.89,122.31,122.66,119.88,113.21 \text { (Ar-C). }\end{array}$ \\
\hline 21 & $\begin{array}{l}2357.71 \mathrm{~cm}^{-1} \text { (Aromatic ring) } 1661.58 \mathrm{~cm}^{-1} \\
(\mathrm{C}=\mathrm{O} \text { conjugated carbonyl group) } \\
1586.90 \mathrm{~cm}^{-1}(\mathrm{C}=\mathrm{C} \text { conjugated }), 1094.27 \\
\mathrm{~cm}^{-1}(\mathrm{C}-\mathrm{Cl} \text { bond })\end{array}$ & $\begin{array}{l}{ }^{1} \mathrm{H} \text { NMR: } 7.994-7.453(\mathrm{~m}, 8 \mathrm{H}, \mathrm{Ar}-\mathrm{H}), 7.408(\mathrm{~d}, 1 \mathrm{H} \text {, ethylenic }), 7.349(\mathrm{~d}, 1 \mathrm{H}, \\
\text { ethylenic }), 6.818(\mathrm{~d}, 1 \mathrm{H} \text {, furyl proton }), 6.770(\mathrm{~d}, 1 \mathrm{H} \text {, furyl proton }),{ }^{13} \mathrm{C} \text { NMR: } 188.39 \\
(\mathrm{C}=\mathrm{O}), 130.72,128.27(\mathrm{C}=\mathrm{C}) 155.45,151.24,139.17,136.55,134.50,129.83,129.16 \text {, } \\
128.94,128.21,125.71,119.22,118.35,114.98,108.69 \text { (Ar-C). }\end{array}$ \\
\hline 22 & $\begin{array}{l}2361.19 \mathrm{~cm}^{-1} \text { (Aromatic ring) } 1659.96 \mathrm{~cm}^{-1} \\
(\mathrm{C}=\mathrm{O} \text { conjugated carbonyl group), } \\
1599.82 \mathrm{~cm}^{-1}(\mathrm{C}=\mathrm{C} \text { conjugated }), \\
1092.15 \mathrm{~cm}^{-1}(\mathrm{C}-\mathrm{Cl} \text { bond }), 1518.53 \mathrm{~cm}^{-1} \\
\left.\text { and } 1357.69 \text { (Asym and sym }-\mathrm{NO}_{2}\right) .\end{array}$ & $\begin{array}{l}{ }^{1} \mathrm{H} \text { NMR: } 7.982-7.431(\mathrm{~m}, 8 \mathrm{H}, \mathrm{Ar}-\mathrm{H}), 7.402(\mathrm{~d}, 1 \mathrm{H} \text {, ethylenic }), 7.319(\mathrm{~d}, 1 \mathrm{H}, \\
\text { ethylenic }), 6.801(\mathrm{~d}, 1 \mathrm{H} \text {, furyl proton }), 6.782(\mathrm{~d}, 1 \mathrm{H} \text {, furyl proton }),{ }^{13} \mathrm{C} \text { NMR: } 188.15 \\
(\mathrm{C}=\mathrm{O}), 131.91,127.95(\mathrm{C}=\mathrm{C}) 152.44,150.54,139.33,136.33,130.06,129.89,129.61 \text {, } \\
129.30,128.99,128.95,128.15,123.99,123.13,119.68,118.03,112.32(\text { Ar-C). }\end{array}$ \\
\hline 23 & $\begin{array}{l}2368.02 \mathrm{~cm}^{-1} \text { (Aromatic ring) } 1662.07 \mathrm{~cm}^{-1} \\
(\mathrm{C}=\mathrm{O} \text { conjugated carbonyl group), } \\
1587.94 \mathrm{~cm}^{-1}(\mathrm{C}=\mathrm{C} \text { conjugated }) 1558.07 \\
\left.\mathrm{~cm}^{-1} \text { and } 1330.55 \text { (Asym and sym }-\mathrm{NO}_{2}\right) \\
1107.92 \mathrm{~cm}^{-1}(\mathrm{C}-\mathrm{Cl} \text { bond }), 1028.14 \mathrm{~cm}^{-1} \\
(\mathrm{C}-\mathrm{Br} \text { bond). }\end{array}$ & $\begin{array}{l}\left.{ }^{1} \mathrm{H} \text { NMR: } 7.953-7.365(\mathrm{~m}, 8 \mathrm{H}, \mathrm{Ar}-\mathrm{H}), 7.340 \mathrm{~d}, 1 \mathrm{H} \text {, ethylenic }\right), 6.980(\mathrm{~d}, 1 \mathrm{H} \text {, ethylenic }) \text {, } \\
7.523(\mathrm{~d}, 1 \mathrm{H} \text {, furyl proton }), 6.415\left(\mathrm{~d}, 1 \mathrm{H} \text {, furyl proton); }{ }^{13} \mathrm{C} \text { NMR: } 189.15(\mathrm{C}=\mathrm{O}),\right. \\
130.70,128.94(\mathrm{C}=\mathrm{C}), 153.70,151.28,139.17,135.45,133.67,132.09,129.82,125.93 \text {, } \\
122.69,119.20,118.40,108.78(\mathrm{Ar}-\mathrm{C}) .\end{array}$ \\
\hline 24 & $\begin{array}{l}2361.19 \mathrm{~cm}^{-1} \text { (Aromatic ring) } 1655.45 \mathrm{~cm}^{-1} \\
(\mathrm{C}=\mathrm{O} \text { conjugated carbonyl group), } \\
1585.17 \mathrm{~cm}^{-1}(\mathrm{C}=\mathrm{C} \text { conjugated }), 1089.21 \\
\mathrm{~cm}^{-1}(\mathrm{C}-\mathrm{Cl} \text { bond }), 1565.32 \mathrm{~cm}^{-1} \text { and } \\
\left.1365.70 \text { (Asym and sym }-\mathrm{NO}_{2}\right)\end{array}$ & $\begin{array}{l}{ }^{1} \mathrm{H} \text { NMR: } 7.999-7.346(\mathrm{~m}, 8 \mathrm{H}, \mathrm{Ar}-\mathrm{H}), 7.295(\mathrm{~d}, 1 \mathrm{H} \text {, ethylenic }), 7.269(\mathrm{~d}, 1 \mathrm{H}, \\
\text { ethylenic }), 7.235(\mathrm{~d}, 1 \mathrm{H} \text {, furyl proton }), 6.860(\mathrm{~d}, 1 \mathrm{H} \text {, furyl proton }),{ }^{13} \mathrm{C} \text { NMR: } 188.44 \\
(\mathrm{C}=\mathrm{O}), 131.03,127.05(\mathrm{C}=\mathrm{C}), 152.75,150.84,139.19,136.57,130.96,130.75,129.85 \text {, } \\
129.23,128.95,128.43,128.31,118.93,118.78,113.91 \text { (Ar-C). }\end{array}$ \\
\hline 25 & $\begin{array}{l}2362.03 \mathrm{~cm}^{-1} \text { (Aromatic ring) } 1665.37 \mathrm{~cm}^{-1} \\
(\mathrm{C}=\mathrm{O} \text { conjugated carbonyl group) } \\
1603.77 \mathrm{~cm}^{-1}(\mathrm{C}=\mathrm{C} \text { conjugated }), 1091.96 \\
\mathrm{~cm}^{-1}(\mathrm{C}-\mathrm{Cl} \text { bond })\end{array}$ & $\begin{array}{l}{ }^{1} \mathrm{H} \text { NMR: } 8.009-7.305(\mathrm{~m}, 8 \mathrm{H}, \mathrm{Ar}-\mathrm{H},), 7.283(\mathrm{~d}, 1 \mathrm{H} \text {, ethylenic }), 7.139(\mathrm{~d}, 1 \mathrm{H}, \\
\text { ethylenic }), 6.820(\mathrm{~d}, 1 \mathrm{H} \text {, furyl proton }), 6.799(\mathrm{~d}, 1 \mathrm{H} \text {, furyl proton }){ }^{13} \mathrm{C} \text { NMR: } 187.40 \\
(\mathrm{C}=\mathrm{O}), 130.26,128.35(\mathrm{C}=\mathrm{C}), 154.09,151.24,137.98,136.14,133.93,131.17,130.84 \text {, } \\
130.30,128.86,123.82,122.93,119.94,118.75,110.52 \text { (Ar-C). }\end{array}$ \\
\hline 26 & $\begin{array}{l}2361.78 \mathrm{~cm}^{-1} \text { (Aromatic ring) } 1651.46 \mathrm{~cm}^{-1} \\
(\mathrm{C}=\mathrm{O} \text { conjugated carbonyl group), } \\
1586.88 \mathrm{~cm}^{-1}(\mathrm{C}=\mathrm{C} \text { conjugated }), 1098.52 \\
\mathrm{~cm}^{-1}(\mathrm{C}-\mathrm{Cl} \text { bond }), 2981.28 \mathrm{~cm}^{-1}(-\mathrm{OH} \\
\text { bond })\end{array}$ & $\begin{array}{l}{ }^{1} \mathrm{H} \text { NMR: } 7.894-7.241(\mathrm{~m}, 6 \mathrm{H}, \mathrm{Ar}-\mathrm{H}), 7.369(\mathrm{~d}, 1 \mathrm{H} \text {, ethylenic }), 7.340(\mathrm{~d}, 1 \mathrm{H}, \\
\text { ethylenic }), 7.225(\mathrm{~d}, 1 \mathrm{H} \text {, furyl proton }), 6.843(\mathrm{~d}, 1 \mathrm{H} \text {, furyl proton }),{ }^{13} \mathrm{C} \text { NMR: } 187.32 \\
(\mathrm{C}=\mathrm{O}), 129.44,128.76(\mathrm{C}=\mathrm{C}), 150.52,149.41,148.56,137.86,130.68,130.47,130.26 \text {, } \\
129.91,128.67,126.74,123.24,122.71,120.42,117.66,110.13,109.03 \text { (Ar-C). }\end{array}$ \\
\hline 27 & $\begin{array}{l}2361.88 \mathrm{~cm}^{-1} \text { (Aromatic ring) } 1659.89 \mathrm{~cm}^{-1} \\
(\mathrm{C}=\mathrm{O} \text { conjugated carbonyl group), } \\
1595.91 \mathrm{~cm}^{-1}(\mathrm{C}=\mathrm{C} \text { conjugated }), 1072.98 \\
\mathrm{~cm}^{-1}(\mathrm{C}-\mathrm{Cl} \text { bond }), 1555.77 \mathrm{~cm}^{-1} \text { and } \\
\left.1350.49 \text { (Asym and sym }-\mathrm{NO}_{2}\right)\end{array}$ & $\begin{array}{l}{ }^{1} \mathrm{H} \text { NMR: } 8.582(\mathrm{~d}, 1 \mathrm{H}, \mathrm{Ar}-\mathrm{H}), 8.190-7.463(\mathrm{~m}, 7 \mathrm{H}, \mathrm{Ar}-\mathrm{H},), 7.354(\mathrm{~d}, 1 \mathrm{H}, \text { ethylenic }) \text {, } \\
6.997(\mathrm{~d}, 1 \mathrm{H} \text {, ethylenic), } 6.940(\mathrm{~d}, 1 \mathrm{H} \text {, furyl proton }), 6.863(\mathrm{~d}, 1 \mathrm{H} \text {, furyl proton); } \\
{ }^{13} \mathrm{C} \text { NMR: } 187.69(\mathrm{C}=\mathrm{O}), 130.24,127.25(\mathrm{C}=\mathrm{C}), 153.29,151.72,148.56,136.44 \text {, } \\
131.86,130.92,130.74,130.66,130.43,130.35,123.89,122.91,119.84,119.29 \text {, } \\
118.58,110.81 \text { (Ar-C). }\end{array}$ \\
\hline 28 & $\begin{array}{l}2360.00 \mathrm{~cm}^{-1} \text { (Aromatic ring) } 1648.68 \mathrm{~cm}^{-1} \\
(\mathrm{C}=\mathrm{O} \text { conjugated carbonyl group), } \\
1585.81 \mathrm{~cm}^{-1}(\mathrm{C}=\mathrm{C} \text { conjugated }), 1028.40 \\
\mathrm{~cm}^{-1}(\mathrm{C}-\mathrm{Br} \text { bond })\end{array}$ & $\begin{array}{l}{ }^{1} \mathrm{H} \text { NMR: } 7.914(\mathrm{~d}, 1 \mathrm{H}, \mathrm{Ar}-\mathrm{H}), 7.651-7.406(\mathrm{~m}, 6 \mathrm{H}, \mathrm{Ar}-\mathrm{H}), 7.241(\mathrm{~d}, 1 \mathrm{H} \text {, ethylenic }) \text {, } \\
6.817(\mathrm{~d}, 1 \mathrm{H} \text {, ethylenic }), 6.782(\mathrm{~d}, 1 \mathrm{H} \text {, furyl proton }), 6.600(\mathrm{~d}, 1 \mathrm{H} \text {, furyl proton }) \\
{ }^{13} \mathrm{C} \text { NMR: } 187.54(\mathrm{C}=\mathrm{O}), 130.38,127.19(\mathrm{C}=\mathrm{C}), 154.68,151.03,136.51,131.93 \text {, } \\
\text { 131.84, } 128.40,126.30,121.87,120.16,118.36,110.00 \text { (Ar-C). }\end{array}$ \\
\hline 29 & $\begin{array}{l}2365.82 \mathrm{~cm}^{-1} \text { (Aromatic ring) } 1653.92 \mathrm{~cm}^{-1} \\
(\mathrm{C}=\mathrm{O} \text { conjugated carbonyl group), } \\
1589.92 \mathrm{~cm}^{-1}(\mathrm{C}=\mathrm{C} \text { conjugated }), 1527.82 \\
\left.\mathrm{~cm}^{-1} \text { and } 1350.38 \text { (Asym and sym }-\mathrm{NO}_{2}\right) \\
1090.65 \mathrm{~cm}^{-1} \text { (C-Cl bond). }\end{array}$ & $\begin{array}{l}{ }^{1} \mathrm{H} \text { NMR: } 8.855(\mathrm{~s}, 1 \mathrm{H}, \mathrm{Ar}-\mathrm{H}), 8.440(\mathrm{~d}, 1 \mathrm{H}, \mathrm{Ar}-\mathrm{H}), 8.373(\mathrm{~d}, 1 \mathrm{H}, \mathrm{Ar}-\mathrm{H}), 7.729(\mathrm{t}, 1 \mathrm{H}, \\
\text { Ar-H), } 7.651(\mathrm{~d}, 1 \mathrm{H}, \mathrm{Ar}-\mathrm{H}), 7.477(\mathrm{~d}, 1 \mathrm{H}, \mathrm{Ar}-\mathrm{H}), 7.425(\mathrm{~d}, 1 \mathrm{H}, \mathrm{Ar}-\mathrm{H}), 7.404(\mathrm{~d}, 1 \mathrm{H}, \\
\text { Ar-H), } 7.345(\mathrm{~d}, 1 \mathrm{H}, \text { ethylenic }), 7.241(\mathrm{~d}, 1 \mathrm{H}, \text { ethylenic }), 6.890(\mathrm{~d}, 1 \mathrm{H}, \text { furyl proton }), \\
6.800(\mathrm{~d}, 1 \mathrm{H}, \text { furyl proton }){ }^{13} \mathrm{C} \text { NMR: } 186.90(\mathrm{C}=\mathrm{O}), 130.65,127.22(\mathrm{C}=\mathrm{C}), 154.98, \\
150.95,148.21,138.79,134.63,133.33,131.20,129.11,128.97,128.04,126.20, \\
125.22,122.64,120.87,110.12,109.11(\text { Ar-C). }\end{array}$ \\
\hline 30 & $\begin{array}{l}2360.97 \mathrm{~cm}^{-1} \text { (Aromatic ring) } 1662.66 \mathrm{~cm}^{-1} \\
(\mathrm{C}=\mathrm{O} \text { conjugated carbonyl group), } \\
1592.11 \mathrm{~cm}^{-1}(\mathrm{C}=\mathrm{C} \text { conjugated), } 1573.89 \\
\left.\mathrm{cm}^{-1} \text { and } 1353.27 \text { (Asym and sym }-\mathrm{NO}_{2}\right)\end{array}$ & $\begin{array}{l}{ }^{1} \mathrm{H} \text { NMR: } 8.838-7.651(\mathrm{~m}, 8 \mathrm{H}, \mathrm{Ar}-\mathrm{H}), 7.616(\mathrm{~d}, 1 \mathrm{H} \text {, ethylenic }), 7.593(\mathrm{~d}, 1 \mathrm{H} \text {, ethy- } \\
\text { lenic), } 7.535(\mathrm{~d}, 1 \mathrm{H} \text {, furyl proton }), 6.989\left(\mathrm{~d}, 1 \mathrm{H} \text {, furyl proton); }{ }^{13} \mathrm{C} \text { NMR: } 186.85\right. \\
(\mathrm{C}=\mathrm{O}), 130.96,127.31(\mathrm{C}=\mathrm{C}), 151.88,150.56,148.16,147.15,138.59,134.27,132.48 \text {, } \\
130.72,130.21,129.03,123.94,122.58,121.59,119.61,118.95,112.96 \text { (Ar-C). }\end{array}$ \\
\hline
\end{tabular}


FTIR analysis: Assignment of selected characteristics IR bands provides significant indication for the formation of chalcones and starting arylfurfurals. In the stating material arylfurfural $(\mathrm{C}=\mathrm{O})$ and $(\mathrm{C}-\mathrm{H})$ absorbed in the expected region; $(\mathrm{C}=\mathrm{O})$ in the $1610-1681 \mathrm{~cm}^{-1}$ and $(\mathrm{C}-\mathrm{H})$ stretch of aldehydes show a weak band in the 2863-2846 $\mathrm{cm}^{-1}$, while in the chalcones $(\mathrm{C}-\mathrm{H})$ stretching band is absent and $(\mathrm{C}=\mathrm{O})$ band shifted to lower wave numbers due to conjugated carbonyl bond and also show absorption band $(\mathrm{CH}=\mathrm{CH})$ in the 1600$1586 \mathrm{~cm}^{-1}$ region which also confirms the formation of chalcones.

${ }^{1} \mathbf{H}$ NMR analysis: The ${ }^{1} \mathrm{H}$ NMR spectra $(300 \mathrm{MHz}$, $\mathrm{CDCl}_{3}$ ) of the staring arylfurfurals show characterstic singlet peak between $\delta$ 9.64-9.72 ppm and two doublet at $\delta 6.78-6.98$ and $\delta 7.29-7.96 \mathrm{ppm}$. The protons belonging to the aromatic ring were observed with the expected chemical shift.

${ }^{13} \mathrm{C}$ NMR analysis: Finally, ${ }^{13} \mathrm{C}$ NMR $\left(75 \mathrm{MHz}, \mathrm{CDCl}_{3}\right)$ spectra of all compounds were recorded and spectral signals are in good agreement with the probable structure. Carbon of $\mathrm{C}=\mathrm{O}$ displayed signal at $\delta 177.21-177.72 \mathrm{ppm}$ in the arylfurfurals while in the chalcones $\mathrm{C}=\mathrm{O}$ carbon displayed signal at $\delta 186.65-198.02 \mathrm{ppm}$ and $\mathrm{CH}=\mathrm{CH}$ shows a singlet at $\delta 121$ and $\delta 137$ ppm respectively.

\section{ACKNOWLEDGEMENTS}

The authors (Samina Aslam, Nadia Asif and Muhammad Nasrullah) would like to acknowledge the financial support by HEC Pakistan in the form of indigenous PhD fellowship and IRSIP grant.

\section{REFERENCES}

1. H.R. Snyder, C.S. Davis, R.K. Brickerton and R.K. Halliday, J. Med. Chem., 10, 807 (1967).

2. W.A. Richards, E. Riss, E.H. Kass and M. Finland, Arch. Int. Med., 96, 437 (1955).

3. B.H. Lipshutz, Chem. Rev., 86, 795 (1986).

4. X.L. Hou, H.Y. Cheung, P.L. Kwan, T.H. Lo, S.Y. Tong and H.N.C. Wong, Tetrahedron, 54, 1955 (1998).

5. B.S. Holla, B.S. Rao, K. Shridhara and P.M. Akberali, IL Farmaco, 55, 338 (2000)
6. W. Hanefeld, M. Schlitzer, N. Debski and H. Euler, J. Heterocycl. Chem., 33, 1143 (1996).

7. A. Krutošíková, J. Kovác and E. Kralovicová, Collect. Czech. Chem. Commun., 48, 772 (1983).

8. R. Kada, D. Ilavský, J. Štetinová, L. Zalibera and J. Padour, Collect. Czech. Chem. Commun., 59, 444 (1994).

9. D. Azanifar and H. Ghasemnejad, Molecules, 8, 642 (2003).

10. M. Lácová, R. Gašparová, D. Loos, T. Liptay and N. Pronayová, Molecules, 5, 167 (2000).

11. R.A. Rane and V.N. Telekar, Bioorg. Med. Chem. Lett., 20, 5681 (2010).

12. M.T. Konieczny, W. Konieczny, M. Sabisz, A. Skladanowski, R. Wakiec, E. Augustynowicz-Kopec and Z. Zwolska, Eur. J. Med. Chem., 42, 729 (2007).

13. D. Kumar, N.M. Kumar, K. Akamatsu, E. Kusaka, H. Harada and T. Ito, Bioorg. Med. Chem. Lett., 20, 3916 (2010).

14. S. Ducki, R. Forrest, J.A. Hadfield, A. Kendall, N.J. Lawrence, A.T. McGown and D. Rennison, Bioorg. Med. Chem. Lett., 8, 1051 (1998); A.M. Asiri and S.A. Khan, Molecules, 16, 523 (2011)

15. R. Edenharder, I.V. Petersdorff and R. Rauscher, Mutat. Res., 287, 261 (1993).

16. S.N. Pandeya, D. Sriram, G. Nath and DeClercq, Eur. J. Med. Chem., 9, 25 (1999).

17. J.S. Biradar, B.S. Sasidhar and R. Parveen, Eur. J. Med. Chem., 45, 4074 (2010).

18. Z. Nowakowska, Eur. J. Med. Chem., 42, 125 (2007).

19. J.M. Devi, K.S. Ali, V.R. Venkatraman, S.K. Ramakrishnan and K. Ramachandran, Thermochim. Acta, 438, 29 (2005).

20. O. Sabzevari, S. Mahmoudian, B. Minaei and H. Paydar, Toxicol. Lett., 196, S213 (2010).

21. B.K. Sarojini, B. Narayana, B.V. Ashalatha, J. Indira and K.G. Lobo, J. Cryst. Growth, 295, 54 (2006).

22. S. Shettigar, G. Umesh, K. Chandrasekharan, B.K. Sarojini and B. Narayana, Opt. Mater., 30, 1297 (2008).

23. B. Delavaux-Nicot, J. Maynadie, D. Lavabre and S. Fery-Forgues, $J$. Organomet. Chem., 692, 874 (2007).

24. K.I. Bhat and M.M.M. Hussain, Asian J. Chem., 21, 3371 (2009).

25. P.S. Patil, S.M. Dharmaprakash, K. Ramakrishna, H.K. Fun, R. Sai, S. Kumar and D.N. Rao, J. Cryst. Growth, 303, 520 (2007).

26. Y. Kumar, R. Green, D.S. Wise, L.L. Wotring and L.B. Townsend, J. Med. Chem., 36, 3849 (1993).

27. M.E. Shoman, M. Abdel-Aziz, O.M. Aly, H.H. Farag and M.A. Morsy, Eur. J. Med. Chem., 44, 3068 (2009).

28. M. Shaharyar, A.A. Siddiqui and M.A. Ali, Bioorg. Med. Chem. Lett., 16, 4571 (2006).

29. B.S. Holla, P.M. Akberali and M.K. Shivandra, IL Farmaco, 56, 910 (2001).

30. C. Rondestvedt, Org. Reactions, 11, 180 (1960). 